\title{
Extensive Performance Evaluation of Efficient Method for Vertical Handoff in Heterogeneous Networks
}

\author{
Surwase S.V. \\ G.H.Raisoni COEM, Pune
}

\author{
Sambare S.S. \\ PCCOE, Pune
}

\begin{abstract}
Handover during mobile communications is becoming the general task in heterogeneous wireless networks. To make handover efficient and faster without leaking data with less delay is prime objective. Since from last two decades, many on-going research studies presented to achieve these goals. Still many research works going on to claim more robust performance. In this paper presenting new method for vertical handover in heterogeneous wireless networks. Taking vertical handoff decision plays significance role. In this paper the extensive simulation studies and performance evaluation for proposed work and comparing it against existing work. Here introduced the fuzzy rule based HMM (FR-HMM) technique for VHD. In addition to this, we further extended FR-HMM method in order to reduce latency and handover delay using concept of automatic authentication auditor (AAA). AAA helps FR-HMM to take speed decision about network selection to perform handover based on logs and history maintained at AAA. Core task of AAA is to minimize the time required for authentication in order to switch to another network using the scores computed for each network. The performance evaluation in done by considering different types of networks to form heterogeneous network such as WIFI, WIMAX, WLAN, UMTS etc. Two different network scenarios are considered one by varying mobility speed and another by varying packet size.
\end{abstract}

\section{Keywords}

WIFI, WIMAX, Vertical Handover Decision, Heterogeneous Networks, AAA, HMM, Fuzzy Rules

\section{INTRODUCTION}

Since from last decade, the next generation communication networks introduced as IP (internet protocol) based networks with use of wireless communication standards such as IEEE 802.11, IEEE 802.15.4, IEEE 802.16, GPRS (General Packet Radio Service), UMTS (Universal Mobile Telecommunication Switching) etc. WIFI is most widely used communication network now days, but it is having limited communication range which makes difficult task for communicating from anywhere anytime. To overcome the limitations of WIFI, UMTS network provides the universal access of network, but having less access rate. Further WIMAX (Worldwide Interoperability for Microwave access) is introduced for wireless communication with high speed and wide range of communication. WIMAX overcomes the limitation of WIFI network and UMTS networks, means covering large area and providing high speed. General idea is to design the hybrid communication network using WIMAX and WIFI with goal of achieving high speed access and wide area of coverage for small to large businesses, smart phones, homes etc. Smartphone's now days are designed to work with different types of networks such as WIMAX, WIFI, 3GPP etc. In order to access the internet services. However the problems of such wireless networks is that they do not tend to handle handovers takes places in wireless network due to the frequent movements of mobile nodes. It becomes worse if network is hybrid means heterogeneous network with combination of two or more wireless networks. In heterogeneous networks, mobile devices cannot directly execute the vertical handover among the various network interfaces as the each network is having different media types and hence incompatible.[2]

To execute vertical handovers in heterogeneous networks, it is required to have efficient method for making VHD. In literature different methods presented for VHD such as using RSS, using SNR, using bandwidths availability, using fuzzy rules etc. The aim of such VHD algorithms is to do the efficient network selection for handover execution so that handover delay and latency should be minimized. All vertical handover methods are having aim of achieving the efficiency in qos (Quality of Service) in mobile communications. In this paper we introduced the novel approach for vertical handover in heterogeneous wireless network based on MIH framework using FR-HMM and AAA model. The Media Independent Handover Services (MIH) is used to solve the problem of compatibility and hence processing the handover processes faster.

The scope of the IEEE802.21 MIH is to develop a standard to enable handover would provide generic link layer intelligence and other network related information from the lower layers (MAC and PHY) to upper layers optimize handoffs between diverse networking technologies viz., 3GPP, 3GPP2 and both wired and wireless media of IEEE802.21. In the mobility management protocol stack of both the MN and network element the MIH Function (MIHF) is a new function located as a shim layer in between the Layer 2 and Layer 3 as shown in Fig. 1.MIHF interacts with different MAC layers of heterogeneous networks, manages them through the MIH service access points (MIH LINK SAP), and exchange information to the upper layer users (MIH SAP), such as MIP, SIP. [3, 4]. However the problems of such wireless networks is that they do not tend to handle handovers takes places in wireless network due to the frequent movements of mobile nodes. It becomes worse if network is hybrid means heterogeneous network with combination of two or more wireless networks. In heterogeneous networks, mobile devices cannot directly execute the vertical handover among the various network interfaces as the each network is having different media types and hence incompatible. 


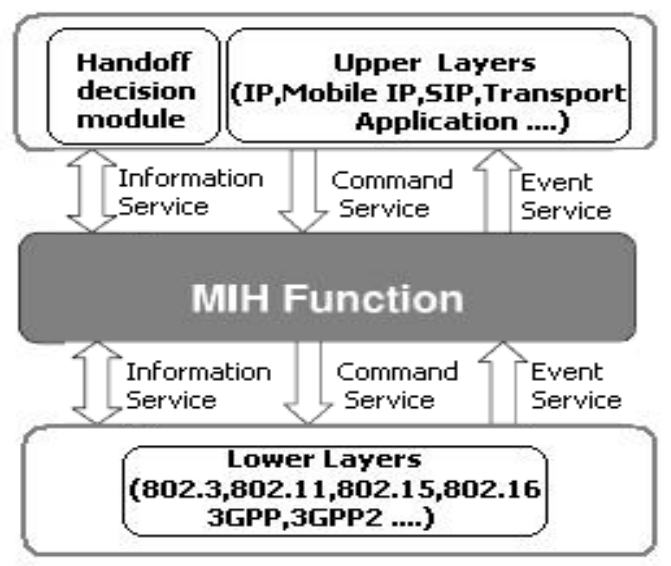

Figure 1: Function of MIH

The performance evaluation of proposed method is done by using NS2 tool for proposed method by using different types of communication data such as audio, video and text. The performance of proposed method is compared against existing FR-HMM and proposed FR-HMM-AAA method in terms of throughput, delay, handover delay etc. In below section of this paper, section 2 discussing about some related methods and techniques proposed for handover in wireless networks. Section 3, discussing the proposed work, algorithms and architecture. Section 4 is discussing about practical results, discussions. Section 5 is presenting the conclusion and future.

\section{LITERATURE SURVEY}

Research on design and implementation of Vertical Handoff Decision (VHD) algorithms has been carried out by many scholars using various techniques. Based on the handoff decision criteria, VHD algorithms are categorized as RSS based algorithms, Bandwidth based algorithms, User Mobility based algorithms and Cost function based algorithms.

\subsection{RSS Based Methods}

In [5], authors proposed the RSS based algorithms in which RSS is the main criteria for decision in this group. Various schemes have been developed to compare RSS of the current point of attachment with that of the candidate point of attachments. They are: Relative RSS, RSS with hysteresis, and RSS with hysteresis plus dwelling timer method. Relative RSS is not applicable for VHD, since RSS from different types of networks cannot be compared directly due to the disparity of the technologies involved. In RSS with hysteresis method, handoff is performed whenever the RSS of new Base station (BS) is higher than the RSS of old BS by a predefined value. In RSS with hysteresis plus dwelling timer method, whenever the RSS of new BS is higher than the RSS of old BS by a predefined hysteresis, a timer is set. When it reaches a certain specified value, handoff is processed. This minimizes Ping pong handoffs. But other criteria have not been considered.

\subsection{Bandwidth Based Methods}

In [6], [7], authors proposed another methods which are based on bandwidth for VHD. In [6], a bandwidth based VHD method is presented between WLANs and a WCDMA network using Signal to Interference and Noise ratio (SINR). It provides higher throughput than RSS based handoffs since the available bandwidth is directly dependent on the SINR. But it may introduce excessive handoffs with the variation of the SINR. These excessive handoffs are reduced by a VHD heuristic based on the Wrong Decision Probability (WDP) prediction [7]. The WDP is calculated by combining the probability of unnecessary and missing handoffs. This algorithm is able to reduce the WDP and balance the traffic load. But in both the papers, RSS is not considered.

\subsection{User Mobility Based Methods}

In [8], [9], authors proposed the user mobility based VHD methods. A handoff to a target network with high bandwidth but weak received signal is not desirable as it may result in breakdown of connection. In user mobility based algorithms, velocity information is a critical one for handoff decision. In the overlay systems, to increase the system capacity, micro/pico cells are assigned for slow moving users and macro cells are assigned for fast moving users by using velocity information. It decreases the number of dropped calls. An improved handoff algorithm [9] has been presented to reduce the number of unnecessary handoffs by using location and velocity information estimated from GSM measurement data of different signal strengths at MS received from base stations. From these papers, it is seen that velocity and location information are also having great effect on handoff management. Therefore they should be taken into account in order to provide seamless handoff between heterogeneous wireless networks.

\subsection{Cost Function Based Methods}

In [10] and [11], authors presented the cost function based algorithms which used the metrics like monetary cost, security, power consumption and bandwidth in a cost function. The handoff decision is made by comparing the result of this function for the candidate networks. Different weights are assigned to different input metrics depending on the network conditions and user preferences.

\subsection{Dynamic Decision Model Method}

In [12], author proposed a dynamic decision model (DDM) to decide the best network at best moment for handoff. The decision model makes the right vertical handoff decisions by determining the best network among available networks based on, the factors such as RSS of network and velocity of mobile station along with static factors like Usage Expense, Link capacity (offered bandwidth) and power consumption. This model not only meets the individual user needs but also improve the whole system performance by reducing the unnecessary handoffs. A trajectory-aware handoff algorithm [13] makes the decision based on position, velocity, signalling delay, and RSS of mobile station. In this algorithm, velocity of MS is divided into two parts as radial velocity and tangential velocity. For more precise handoff initiation, tangential velocity of MS is neglected, and only radial velocity of MT is considered in handoff decision making. Moreover, before handoff decision, least square line method is applied to RSS of MS to avoid unnecessary back-and-forth handoffs (Ping-Pong handoffs) between different services. All the above algorithms have not considered the mobility prediction methods to take proactive measures.

\section{PROPOSED VHD METHOD}

In this proposed handover method going to consider WIMAX, WLAN networks for practical analysis of proposed VHD algorithm. The proposed VHD algorithm is based on fuzzy logic and HMM model. In this section presenting the detailed architecture of proposed approach. Following is the basic algorithm steps for proposed VHD. In addition to this concept of AAA is introduced with below VHD algorithm to optimize the performance of vertical handover. 


\subsection{VHD Algorithm}

Step 1: Currently some application is running on mobile

Step 2: If RSS (Received Signal Strength) is less than RSS threshold then Initialize handover else keep on monitoring RSS for above condition.

Step 3: In handover process collect 1.RSS 2.Data rate 3.Coverage area 4.Bandwidth etc. information of all available networks.
Step 4: According to current user application requirement scores are calculated for each network using fuzzy logic.

Step 5: Check if highest scored network is congested then handover to next high score network else handover to the same network.

Figure 2 below showing the overall flowchart for proposed VHD which is based on fuzzy rules and HMM.

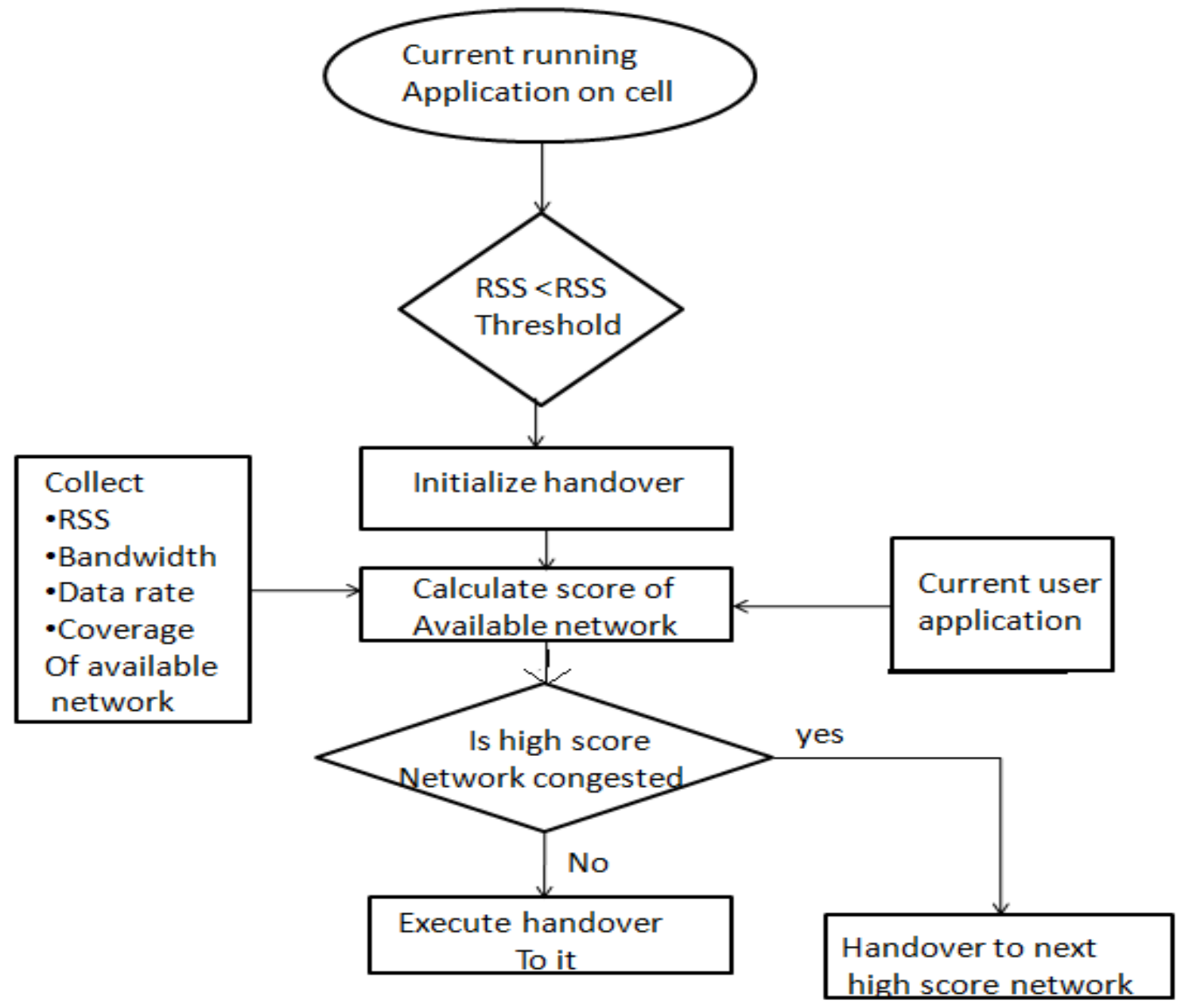

Figure 2: Proposed System Architecture

\subsection{Fuzzy Logic Method}

Fuzzy logic is a process of decision making based on input membership functions and a group of fuzzy rules, like the human brain, which simulates the interpretation of uncertain sensory information. Here, fuzzy logic is applied in order to select the most appropriate network from the list of networks that are available from the scanning phase, which is handled by the MN. Normally, the MN does not know which AP will be a good partner to perform a handover, and it can just depend on the quality of the current and available link. In addition, sometimes, the MN will perform unneeded handovers during its roaming. In other words, the handover obtained by incorrect decision-making will cause overhead signaling and increase the handover latency time. Therefore, fuzzy logic is the answer to this uncertain type of problem.

In proposed fuzzy logic system, the information gathered (RSS, bandwidth, network coverage, Data rate) depending on their availability are fed into a fuzzier in which are converted into fuzzy sets. A fuzzy set contains varying degree of memberships in the sets. The membership values are obtained by mapping the values retrieved for the particular variable into a membership function.

- The input fuzzy variable "RSS" has three fuzzy sets: weak, normal, and strong.

- The input fuzzy variable "bandwidth" has three fuzzy sets: low, normal, and high.

- The input fuzzy variable "network coverage" has three fuzzy sets: bad, normal, and good.

- The input fuzzy variable "Data rate" has three fuzzy sets: less, normal, and more.

These inputs are chosen answering specific needs related to different scenarios. RSS indicates the current radio link quality and acts as a pretreatment that helps to decide whether to trigger the handover. The bandwidth is different from a network to another (e.g., 3G has lower bandwidth compared to WLAN). 


\subsection{HMM Prediction Model}

In the proposed work, a HMM based prediction model is executed by each BS or AP in the network. Let us assume that each BS holds the list of their location and other BSs. All of the user movements are in the hidden states. The observed states are obtained from the received signal strength (RSS) values are sent by every mobile node. The BS in which the mobile is currently residing is in the home BS. The home BS predicts the list of possible BSs in the movement of a direction of the mobile station.

\subsection{Third Party Auditor (AAA) Algorithm}

Elements needed in the Reinforcement learning will be agent, environment along with four main sub-elements of the system: a policy, a reward function, a value function, and, optionally, a model of the environment. For proposed vertical handoff algorithm, system model is assumed to be a heterogeneous integrated system of various networks like GSM, UMTS, LTE, WiMax, Wi-Fi represented by Network 1, Network 2, Network 3, Network 4 and Network 5.

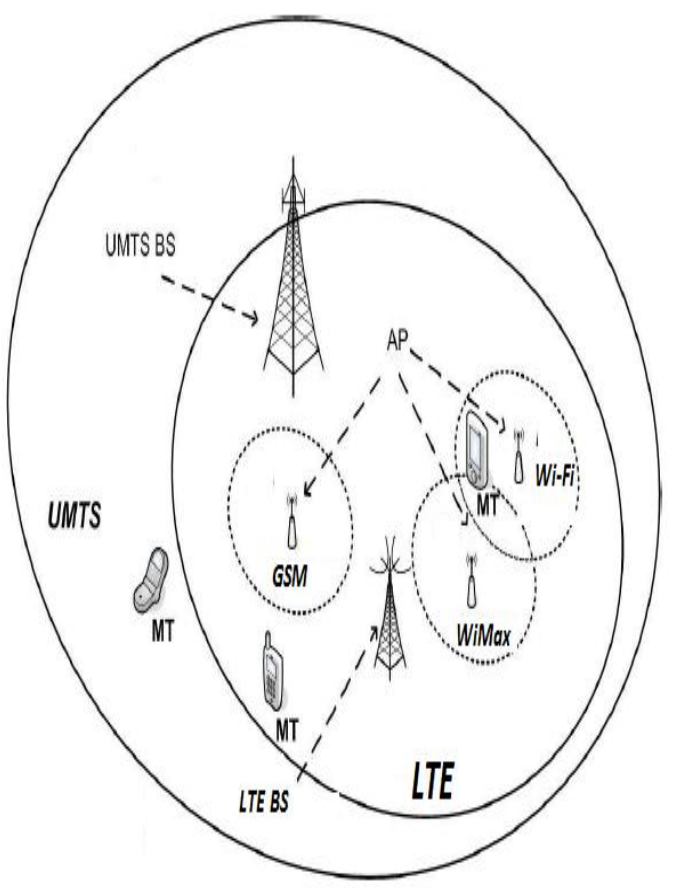

Figure 3: Example of Heterogeneous Network

During the time of connection the mobile terminal will continuously scan the coverage area of the current network. As it is observed that the coverage area of the current network is less than a threshold it will scan the adjacent networks having coverage area for handover. During each handover decision, the mobile terminal has to choose an action (i.e., select a network) based on the current application and previous history of delay of the target networks.

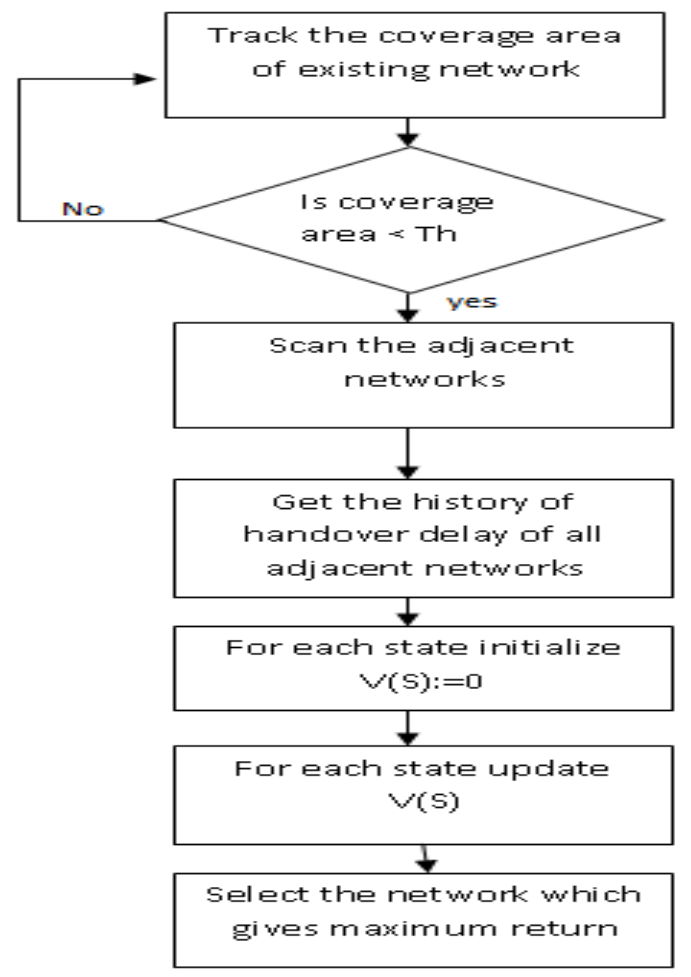

Figure 4: Working of AAA method

With this state and action, the system then evolves to a new state according to a transition probability function. This new state lasts for a period of time until the next handover decision comes, and then the Mobile Terminal (MT) makes a new decision again (i.e., selects a network again). For any action that the Mobile Terminal chooses at each state, there is a reward and a discount factor associated with it.

The goal of each MT is to maximize the expected total reward that it can obtain during the handover to the target network.

The goal of the MDP is to choose a policy that will maximize the return at any start state.

With a policy $\pi: \mathrm{S} \rightarrow \mathrm{A}$, the discounted reward function is given as [11],

$$
V \pi(S)=E\left[\sum_{k=1}^{D} \gamma k R(s k, a k) \mid s 1=s\right]
$$

Where,

$$
\begin{aligned}
& V \pi(S)-\text { The expected return starting at state } \\
& \text { S following } \pi \\
& \gamma k-\quad \text { Discount factor } \\
& \text { D - } \quad \text { Length of MDP }
\end{aligned}
$$

The reward function is the expected sum of rewards that can be obtained through the process when starting from state $S$. The above equation can be rewritten in the form of Bellman equation as follows:

$$
V(S)=\max _{a \in A}\left[R(s, a)+\gamma \sum P s a\left(s^{\prime}\right) V\left(S^{\prime}\right)\right]
$$

Where $P s a\left(s^{\prime}\right)$ is the probability of transitioning from state s to $S^{\prime}$ ' when action is taken. 


\section{PRACTICAL RESULTS AND ANALYSIS}

This simulation study is done using NS2. For practical analysis of proposed VHD algorithm we used below listed networks:

\section{WIMAX}

WIFI

WLAN

GSM

LTE

UMTS

The network is composed of this all wireless networks for the evaluation of proposed work. The application considers here are audio, video and text.

Case 1 Result: Varying Mobility Speed for Different Applications of communication.

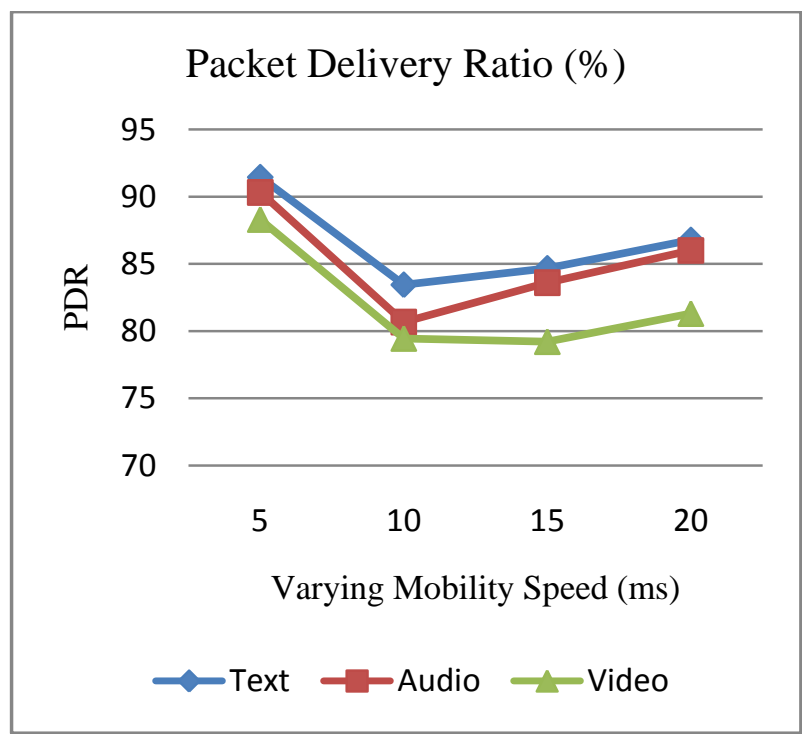

Figure 5: PDR Performance Analysis

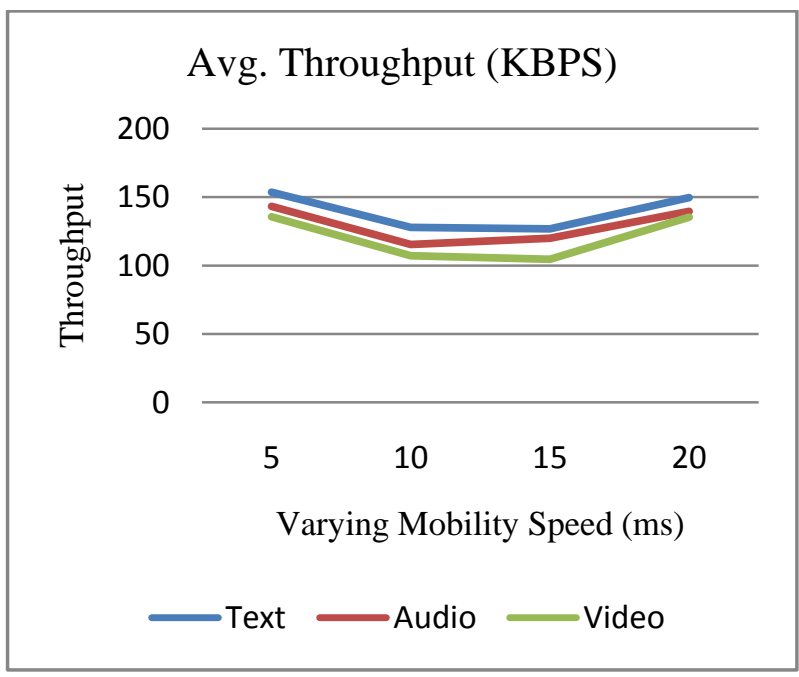

Figure 6: Throughput Performance Analysis

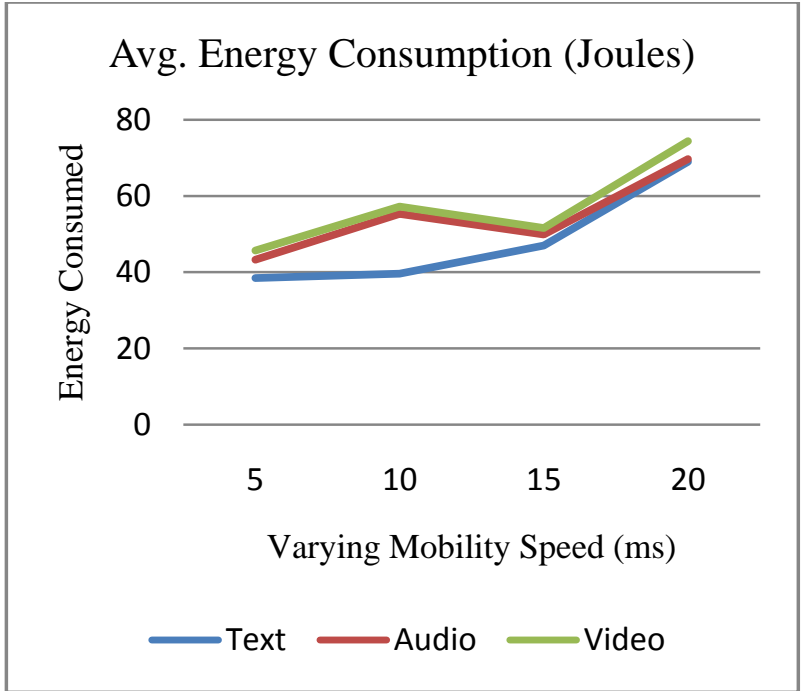

Figure 7: Energy consumption Performance Analysis

Above results in figures 5, 6 and 7 are showing the performance analysis for PDR, throughput and average energy consumption using proposed method of VHD. These results indicating that there is no much difference in performance application wise. Generally in existing cases, performance for audio/video applications is very worst, but here we have achieved it near to text applications.

\section{Case 2 Results: Varying Packet Size}

Varying packet size to check the performance of proposed method against existing method for text application communication

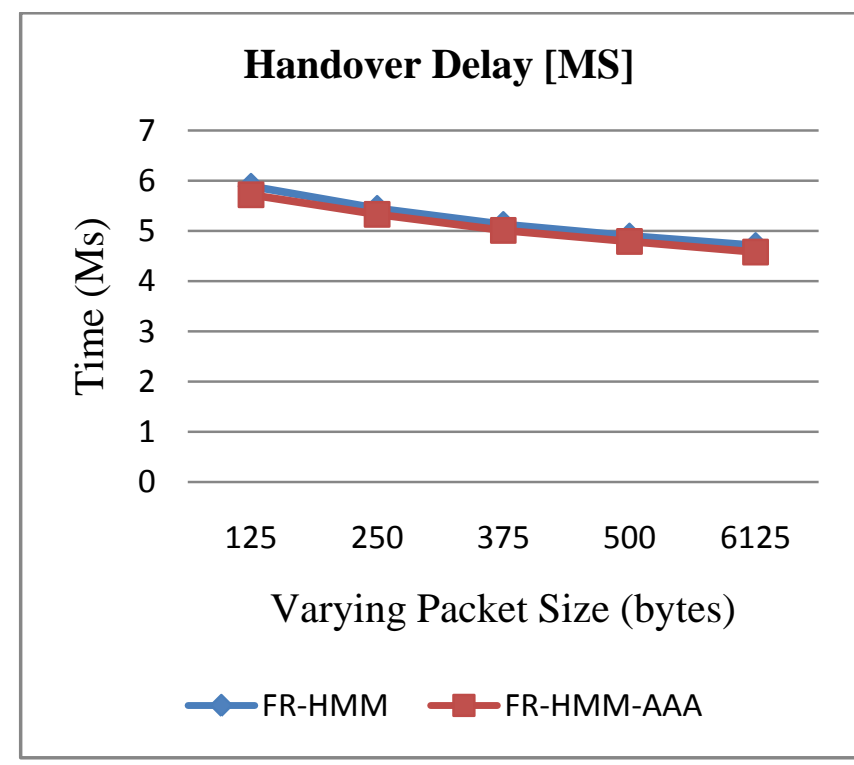

Figure 8: Handover Delay Performance Analysis 


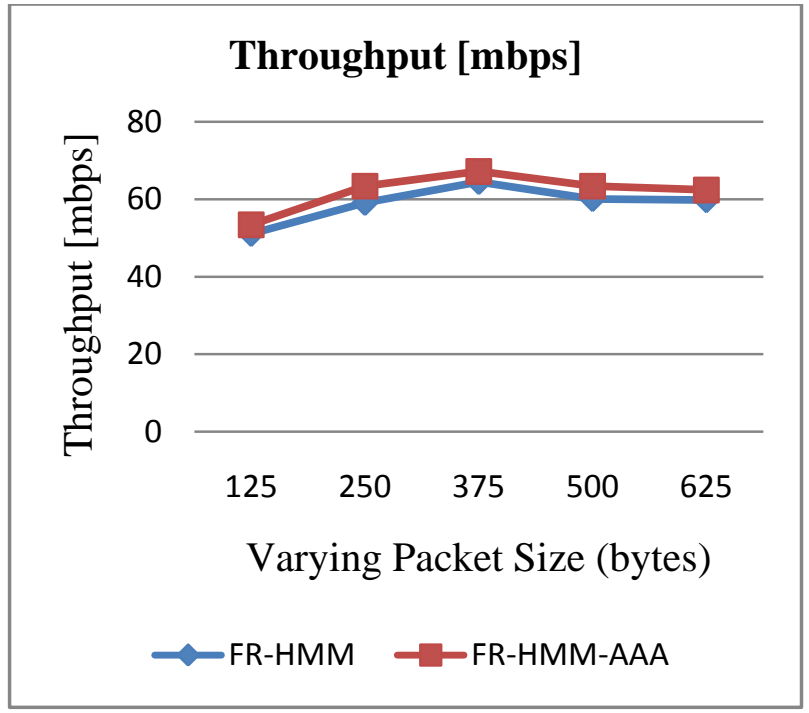

Figure 9: Throughput Performance Analysis

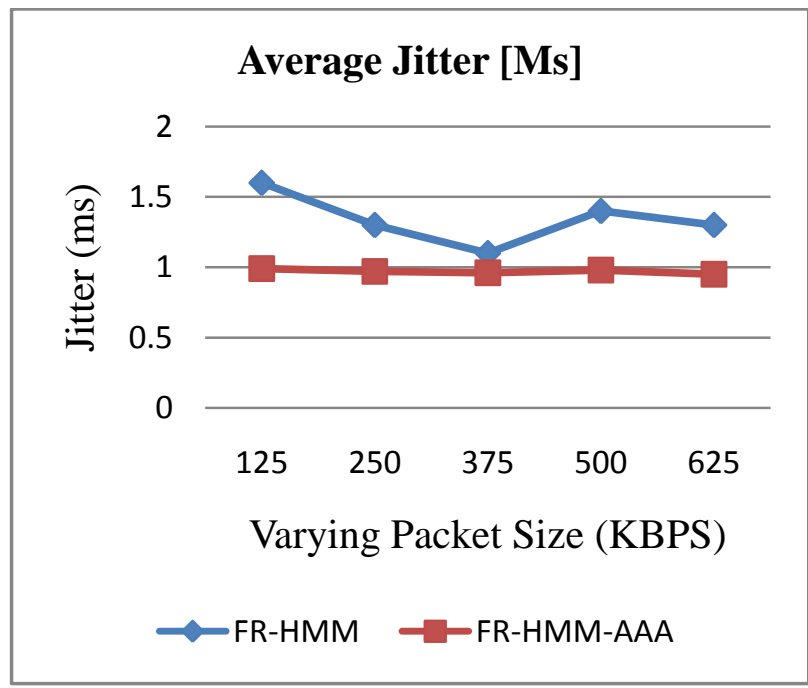

Figure 10: Average Jitter Performance Analysis

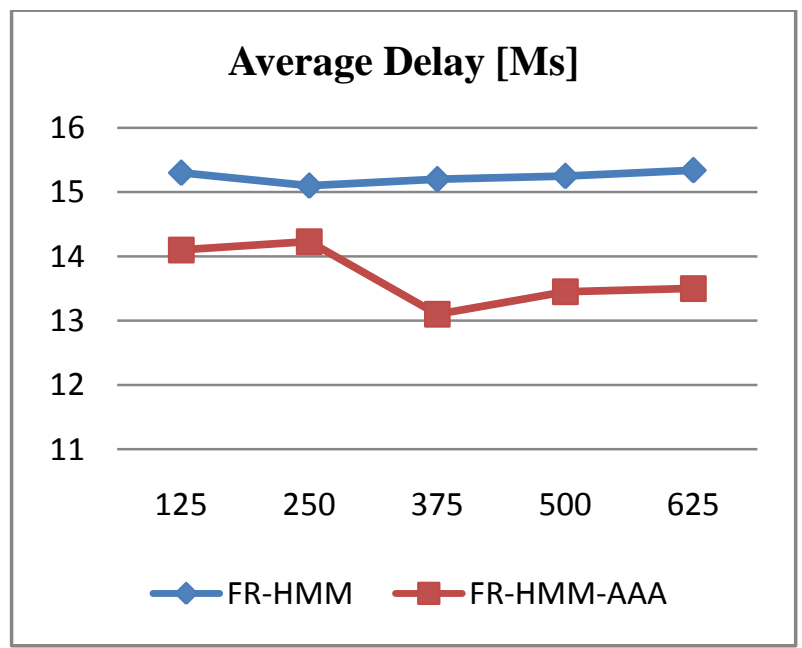

Figure 11: Average Delay Performance

Figures from 8-11 are showing the performance results for proposed method and existing method with varying packet sizes. From these results, it is showing that, due to the use of AAA in FR-HMM, handover delay, jitter is minimized and hence throughput performance and average delay performance is improved as compared to existing FR-HMM method of VHD.

\section{CONCLUSION AND FUTURE WORK}

In this paper new method called Fuzzy based HMM with AAA VHD proposed. The FR-HMM predictor has been used which accurately estimates the next location visited by a mobile user, the received signal strength values of the MNs. The main goal of using AAA with existing FR-HMM is to save the authentication time and handover time. Practical analysis is showing the results for both FR-HMM and FRHMM-AAA. The performance of such methods is evaluated using different kinds of networks together with varying mobility speed with different communication applications and varying packet size. Proposed method is outperforming existing method for all performance metrics. Future work will be on improving the scalability and reliability of proposed method if number of mobile nodes and handovers are more in network.

\section{REFERENCES}

[1] Ken Murray,Rajiv Mathur \& Dirk Pesch, "Intelligent Access and Mobility Management in Heterogeneous Wireless Network using policy", Adaptive Wireless Systems Group Department of Electronic Engineering Cork Institute of Technology,Cork,Irland.

[2] Jun-Zhao Sun, Douglas Howie, and Jaakko Sauvola, "Mobility management techniques for the next generation wireless networks", MediaTeam, Machine Vision and Media Processing Unit, Infotech Oulu University of Oulu, Finland.

[3] F. Barcelo, "Performance analysis of handoff resource allocation strategies through the state-dependent rejection scheme", IEEE Transactions on Wireless Communications 3 (3) (2004) 900-909.

[4] A.H. Zahran, B. Liang, A. Saleh, "Signal threshold adaptation for vertical handoff in heterogeneous wireless networks", Mobile Networks and Applications 11 (4) (2006) 625-640.

[5] S. Mohanty, I.F. Akyildiz, “A cross-layer (layer $2+3$ ) handoff management protocol for next-generation wireless systems", IEEE Transactions on Mobile Computing 5 (10) (2006) 1347-1360.

[6] Mr. Musab Q. Al-Ghadi. Dr. Ismail M. Ababneh, Dr. Wail E. Mardini, "Performance study of SINR scheme for Vertical Handoff in wireless networks", in: Proceedings of the 2007 IEEE Global Telecommunications Conference (GLOBECOM'07), Washington, DC, USA, November 2007, pp. 4483-4487.

[7] Elaheh Arabmakki, Sherif Rashad, Member, IEEE, and Sadeta Krijestorac. "An Algorithm for Optimizing Vertical Handoff between WLAN and Cellular Networks", Cyber Journals: Multidisciplinary Journals in Science and Technology, Journal of Selected Areas in Telecommunications (JSAT), August Edition, 2011.

[8] Juang.R.T, H.P.Lin and D.B.Lin,2005, "An improved location-based handover algorithm for GSM systems", Proc of wireless communications and networking conference, Mar.13-17, pp-1371-1376. 
[9] Xiao.C, K.D.Mann and J.C.Olivier,2001, "Mobile speed estimation for TDMA-based hierarchical cellular systems", Proc.Trans. Veh.Technol,50, 981-991.

[10] F. Zhu, J. McNair, "Multiservice vertical handoff decision algorithms", EURASIP Journal on Wireless Communications and Networking 2006 (2) (2006) 52.

[11] N. Nasser, A. Hasswa, H. Hassanein, "Handoffs in fourth generation heterogeneous networks", Communications Magazine 44 (10) (2006) 96-103
[12] N. Nasser, S. Guizani, E. Al-Masri, "Middleware vertical handoff manager: a neural network-based solution", in: Proceedings of the 2007 IEEE International Conference on Communications (ICC'07), Glasgow, Scotland, June 2007, pp. 5671-5676.

[13] Thazin Ei and Furong Wang, "A trajectory-aware handoff algorithm based on GPS information”, Springer Link 2009. 